\title{
Editorial
}

\section{Scheduling Problems with Due Date Assignment}

\author{
Yunqiang Yin, ${ }^{1}$ Shuenn-Ren Cheng, ${ }^{2}$ John Y. Chiang, ${ }^{3}$ Jason C. H. Chen, ${ }^{4}$ \\ Xuerong Mao, ${ }^{5}$ and Chin-Chia $\mathrm{Wu}^{6}$ \\ ${ }^{1}$ Faculty of Management and Economics, Kunming University of Science and Technology, Kunming 650093, China \\ ${ }^{2}$ Graduate Institute of Business Administration, Cheng Shiu University, Kaohsiung, Taiwan \\ ${ }^{3}$ Department of Computer Science \& Engineering, National Sun Yat-Sen University, Kaohsiung, Taiwan \\ ${ }^{4}$ School of Business, Gonzaga University, E 502 Boone Avenue, Spokane, WA 99258, USA \\ ${ }^{5}$ Department of Mathematics and Statistics, University of Strathclyde, UK \\ ${ }^{6}$ Department of Statistics, Feng Chia University, Taichung, Taiwan
}

Correspondence should be addressed to Yunqiang Yin; yunqiangyin@gmail.com

Received 18 March 2015; Accepted 18 March 2015

Copyright (C) 2015 Yunqiang Yin et al. This is an open access article distributed under the Creative Commons Attribution License, which permits unrestricted use, distribution, and reproduction in any medium, provided the original work is properly cited.

Scheduling problems with due date assignment have attracted much research attention in the past few decades due to the introduction of new concepts and practices of operations management. Meanwhile, there are a lot of works by building up a discrete mathematical approach restricted by the absence of general principles that could play the same role as the vibrational principles in operation research or application statistics. The issue on scheduling problems with due date assignment inspires many researchers to address many interesting manuscripts most of which are discussed widely over single-machine, flowshop, or parallel settings.

One manuscript addresses the topic on common due date assignment over a parallel-batching machine. H. Gong et al. consider the parallel-batching scheduling problem with delivery involving both batching scheduling and common due date assignment. They prove that the problem is NPhard in the strong sense and build dynamic programming algorithms for two special cases with a given sequence of orders on the machine and a given batching in the production part, respectively. Three manuscripts concentrate on two-agent scheduling on single-machine setting. L. Wan investigates a two-agent scheduling problem on a single machine to minimize the maximum cost with positiondependent jobs. In the problem setting, the actual positiondependent processing time of one job is characterized by variable function dependent on the position of the job in the sequence. The author develops a feasible method to achieve all the Pareto optimal points in polynomial time. P. Liu and L. Duan consider a scheduling problem with resource dependent release times and two agents. Their objective is to find a schedule for the problem of minimizing A-agent's total amount of resource consumption with a constraint on Bagent's makespan. They propose a polynomial time algorithm to solve the problem. S. Liu et al. consider a two-agent scheduling on a single machine to minimize the A-agent's total completion time with the restriction that has an upper bound on B-agent's total completion time. They use a branchand-bound and three simulated annealing algorithms to solve the proposed problem. Five manuscripts focus on new developments in scheduling approach in operation research. Q. Liu et al. consider the online scheduling problem on a single machine with the assumption that all jobs have their processing times in specified interval and all the jobs should be first processed on a single machine and then delivered by a vehicle to some customer. Public transit providers are often facing continuous pressure to improve service quality and reduce operating costs. Inspired by this observation, $\mathrm{H}$. $\mathrm{Li}$ et al. address a bus driver scheduling problem. Sometime real-life large and complex problem instances often need new approaches to overcome the computational difficulties in solving them, and thus they propose a column generation based hyperheuristic for finding near-optimal solutions. Ship block construction space is an important bottleneck resource in the process of shipbuilding, so the production scheduling optimization is a key technology to improve the efficiency of shipbuilding. S. Hu et al. propose a hybrid heuristic algorithm for the ship block construction space scheduling problem. According to different driving behavioral characteristics of 
bus drivers, Z. Zhang et al. propose a cellular automata traffic model considering the bus lane-changing behavior with scheduling parameters. For a better service level of a train operating plan, W. Zhou et al. address an integrated optimization method of train planning and train scheduling.

Four potential topics in operation research or application statistics include to explore the key influencing factors of lowcarbon development for logistics companies are investigated by using the data from the questionnaire by X. Y. Duan et al., using multiple-decision procedures to test the homogeneity of mean for $k$ exponential distributions by H.-C. Chen et al., discussing a unified weight formula to find the sample variance from weighted successive difference by K.-H. Lo et al., and composite transcendental entire functions with certain gaps which have no unbounded Fatou component by C. Yang and S. Wang.

Finally, we hope these papers may enrich and provide a guide to the readers to treat scheduling with due date assignment or further developments in operation research or application statistics.

Yunqiang Yin

Shuenn-Ren Cheng

John Y. Chiang

Jason C. H. Chen

Xuerong Mao

Chin-Chia Wu 


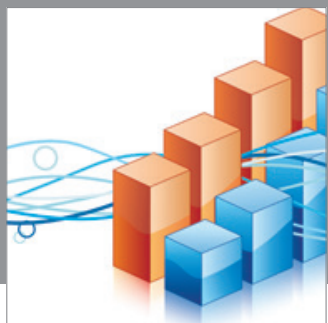

Advances in

Operations Research

mansans

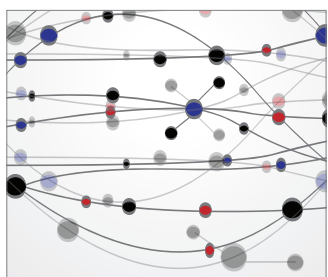

The Scientific World Journal
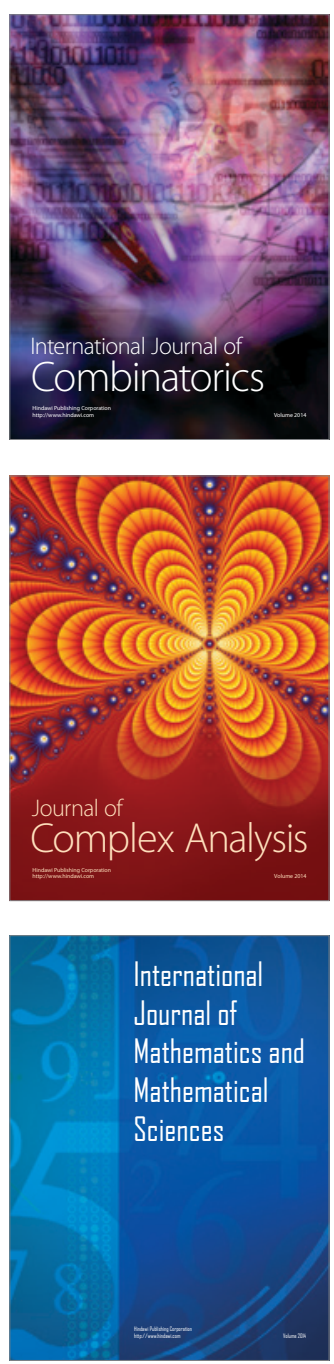
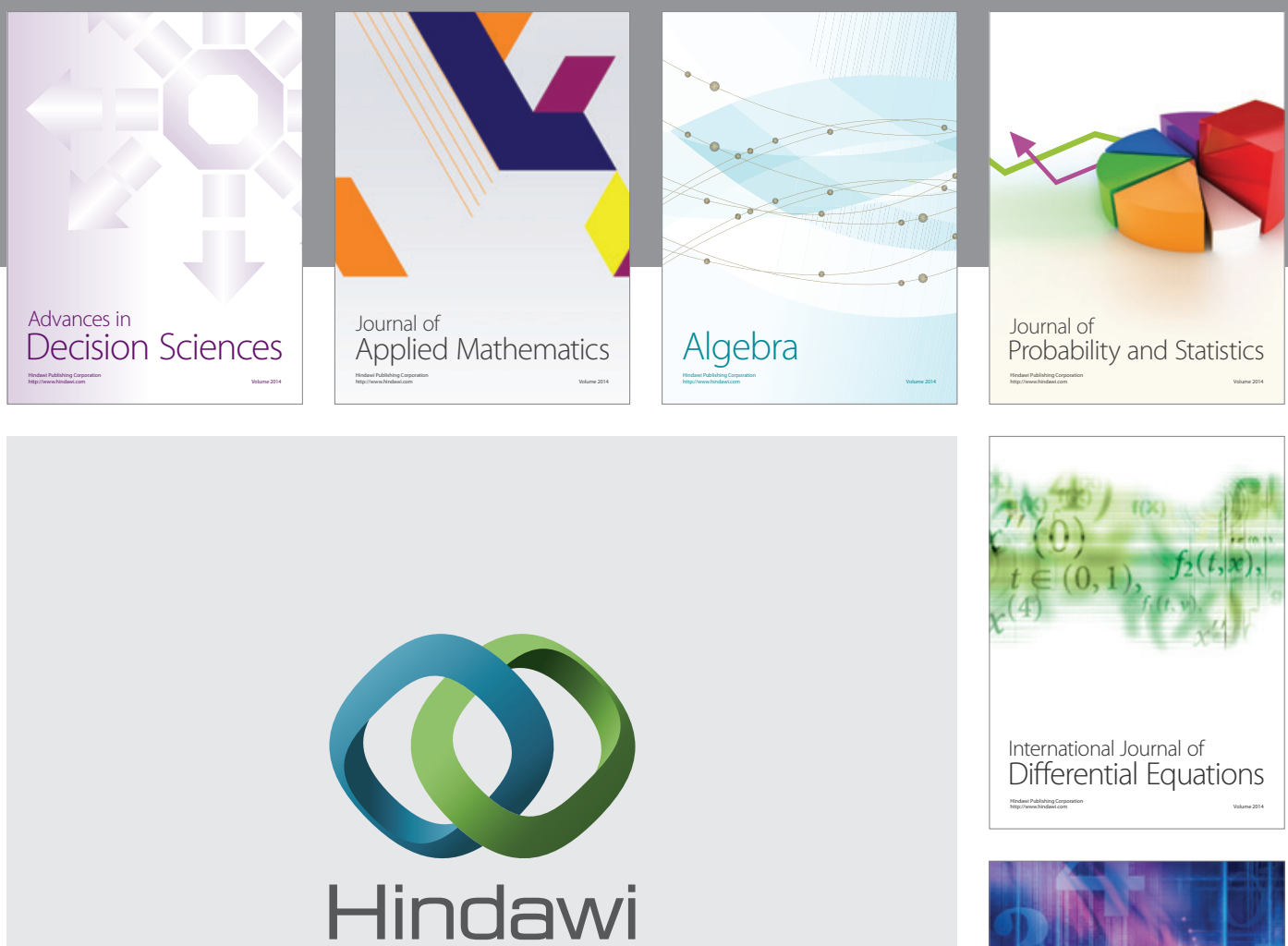

Submit your manuscripts at http://www.hindawi.com
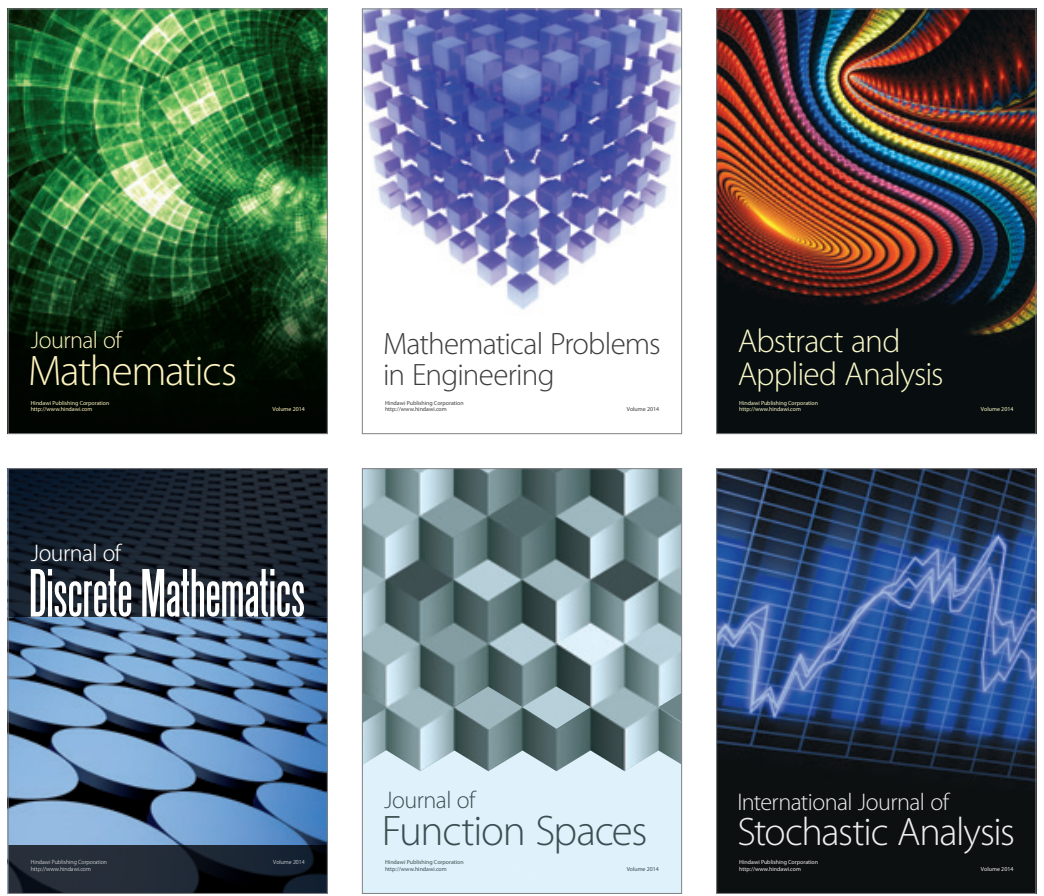

Journal of

Function Spaces

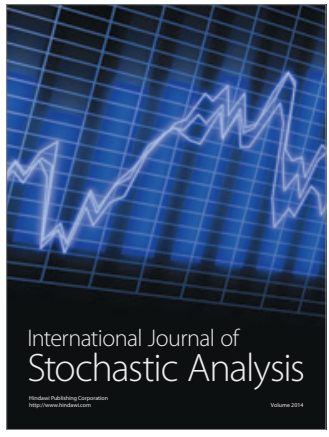

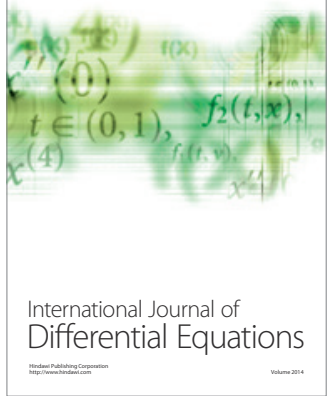
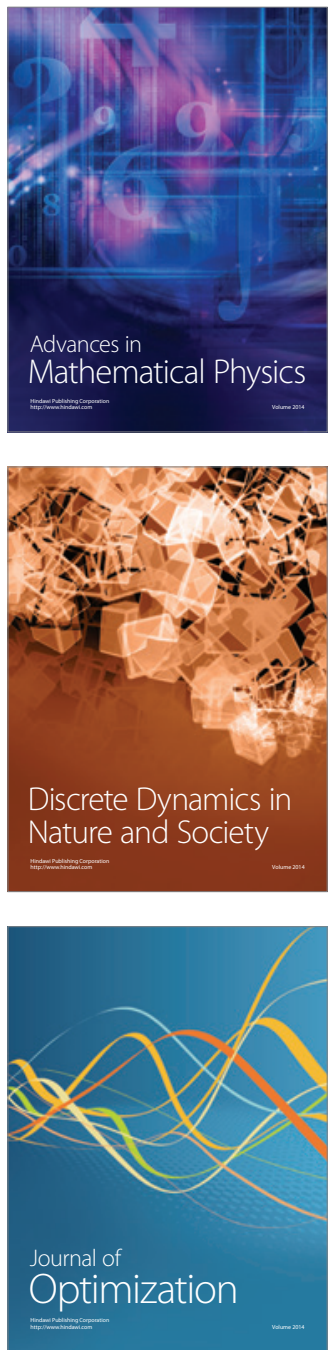